\title{
On moments of the integrated exponential Brownian motion
}

\author{
Francesco Caravelli ${ }^{1,2,3, a}$, Toufik Mansour ${ }^{4}$, Lorenzo Sindoni ${ }^{1}$, and Simone Severini ${ }^{3}$ \\ 1 Invenia Labs, 27 Parkside Place, Cambridge CB1 1HQ, UK \\ 2 London Institute for Mathematical Sciences, 35a South Street, London W1K 2XF, UK \\ 3 University College London, Gower Street, London WC1E 6BT, UK \\ 4 University of Haifa, 31905 Haifa, Israel
}

Received: 28 May 2016

Published online: 27 July 2016

(C) The Author(s) 2016. This article is published with open access at Springerlink.com

\begin{abstract}
We present new exact expressions for a class of moments of the geometric Brownian motion in terms of determinants, obtained using a recurrence relation and combinatorial arguments for the case of a Itô's Wiener process. We then apply the obtained exact formulas to computing averages of the solution of the logistic stochastic differential equation via a series expansion, and compare the results to the solution obtained via Monte Carlo.
\end{abstract}

\section{Introduction}

The geometric Brownian motion is the stochastic process described by the differential equation,

$$
\mathrm{d} f=\mu f \mathrm{~d} t+\sigma f \mathrm{~d} W_{t},
$$

where $W_{t}$ is a Wiener process and $\mu, \sigma$ are constants describing the drift and the variance of the noise, respectively. The solution can be written as

$$
f\left(W_{t}, t\right)=\exp \left\{\left(\mu-\frac{\sigma^{2}}{2}\right) t+\sigma W_{t}\right\} .
$$

Geometric Brownian motion is used for modelling many phenomena in a variety of contexts [1]. A prominent role is played in financial applications, where the distribution of returns can be approximated by a log-normal distribution [1, $2]$, at least in specific regimes. The model is time-reversal asymmetric and implements the multiplicative noise typical of several models and physical systems, both in finance [3] and in biology [4].

For the computation of certain properties, it is however very often necessary to evaluate the integral of $f(W, t)$ over a time interval,

$$
F[W, t]=\int_{0}^{t} f\left(W_{s}, s\right) \mathrm{d} s .
$$

The evaluation of this functional is also involved in the solution of the geometric Brownian motion with logistic corrections as we will see in sect. 3 . In general, averages of the form

$$
\langle G(F[W, t])\rangle=\sum_{k=0}^{\infty} a_{k}\left\langle F[W, t]^{k}\right\rangle \equiv \sum_{k=0}^{\infty} a_{k} r_{k}
$$

are quite common, and thus detailed results are already available in the applied probability $[5,6]$ and the statistical physics literature [7-9].

In this paper, we will derive new exact formulas for the evaluation of these integrals, under the assumption of the Itô formulation for the Wiener process. Similar results have been given in $[2,10]$. Motivated by obtaining exact

\footnotetext{
a e-mail: francesco.caravelli@gmail.com
} 
formulas for Asian options, in [10] Yor obtained an exact formula in terms of polynomials for the following moments:

$$
Y_{n}=\left\langle e^{\sigma W_{t}}\left(\int_{0}^{t} \mathrm{~d} \tilde{t} e^{W_{\tilde{t}}}\right)^{n}\right\rangle .
$$

Using Girsanov's theorem [11], one can derive a series of identities, in which the last is Bougerol's formula

$$
\left\langle e^{\sigma W_{t}}\left(\int_{0}^{t} e^{W_{\tilde{t}}} \mathrm{~d} \tilde{t}\right)^{n}\right\rangle=\left\langle P_{n}\left(e^{2 W_{t}}\right)\right\rangle=4^{n}\left\langle\frac{\sinh \left(W_{t}\right)^{2 n}}{\left\langle W_{1}^{2 n}\right\rangle}\right\rangle,
$$

where

$$
P_{n}(z)=\Gamma(n) \sum_{j=0}^{n} c_{j} z^{j}
$$

and

$$
c_{j}=\prod_{k \neq j 0 \leq k \leq n} \frac{2}{(\mu+j)^{2}-(\mu+k)^{2}} .
$$

Such integrals emerge, for instance, in the description of financial markets when one includes the effect of transaction costs [12-14], i.e. the effect of the trader position on the price. These type of integrals were also studied more recently in [15] using the technique of finite differences [16], in which exact results were derived and provided an application of the Hermite-Genocchi formula.

In this work, we take a different route for evaluating these integrals using combinatorics. Specifically, we provide a new exact formula in terms of a determinant for evaluating these moments. We prove a recurrence relation for the integrals involved at the $k$-th order in terms of integrals at the $(k-1)$-th order, and after resummation, we get an identity in terms of a determinant. We then use these results in the evaluation of the averages for the archetypical logistic stochastic differential equation employed to describe the effect of transaction costs in perturbation theory. The paper is organized as follows. In sect. 2 we derive the main theorems, which are based upon three lemmas. In sect. 3 we apply our formulae to the case of a logistic stochastic different equation solution as a direct application. Conclusions follow.

\section{Calculation of moments}

The central quantity of interest in the present paper is given by the average over the Wiener process $W_{s}$ :

$$
r_{k}(\mu, \sigma, t) \equiv\left\langle F[W, t]^{k}\right\rangle .
$$

If we expand eq. (9), we obtain

$$
\left\langle F[W, t]^{k}\right\rangle=\int_{0}^{t} \mathrm{~d} \tilde{t}_{k} \ldots \int_{0}^{t} \mathrm{~d} \tilde{t}_{1}\left\langle e^{\sum_{i=1}^{k}\left[\left(\mu-\frac{\sigma^{2}}{2}\right) \tilde{t}_{i}+\sigma W_{\tilde{t}_{i}}\right]}\right\rangle .
$$

We will use the following formula due to the properties of integrals with Gaussian measure $[1,17]$, and in which we assume that $\left\langle W_{t} W_{t^{\prime}}\right\rangle$ is of the Itô type. This implies

$$
\begin{aligned}
\left\langle e^{\sigma \sum_{i=1}^{k} W_{\tilde{t}_{i}}}\right\rangle & =e^{\frac{\sigma^{2}}{2} \sum_{i, j=1}^{k}\left\langle W_{\tilde{t}_{i}} W_{\tilde{t}_{j}}\right\rangle} \\
& =e^{\frac{\sigma^{2}}{2} \sum_{i, j=1}^{k} \min \left(\tilde{t}_{i}, \tilde{t}_{j}\right)} .
\end{aligned}
$$

By using this property, we can now prove the following fact:

Lemma 1. For the average over the Wiener process $W_{s}$ of Itô type, the following formula holds true:

$$
\begin{aligned}
r_{k}(\mu, \sigma, t)= & \Gamma(k+1) \int_{0}^{t} e^{\mu \tilde{t}_{k}} \int_{0}^{\tilde{t}_{k-1}} e^{\left(\mu+\sigma^{2}\right) \tilde{t}_{k-2}} \ldots \\
& \ldots \int_{0}^{\tilde{t}_{3}} e^{\left(\mu+(k-2) \sigma^{2}\right) \tilde{t}_{2}} \int_{0}^{\tilde{t}_{2}} e^{\left(\mu+(k-1) \sigma^{2}\right) \tilde{t}_{1}} \mathrm{~d} \tilde{t}_{1} \ldots \mathrm{d} \tilde{t}_{k} .
\end{aligned}
$$


Proof. By a direct application of eq. (11)

$$
\begin{aligned}
r_{k}(\mu, \sigma, t) & =\langle\underbrace{\left.\int_{0}^{t} \ldots \int_{0}^{t} e^{\sum_{i=1}^{k}\left[\left(\mu-\frac{\sigma^{2}}{2}\right) \tilde{t}_{i}+\sigma W_{\tilde{t}_{i}}\right]} \mathrm{d} \tilde{t}_{1} \ldots \mathrm{d} \tilde{t}_{k}\right\rangle}_{k} \\
& =\int_{0}^{t} \ldots \int_{0}^{t} e^{\sum_{i=1}^{k}\left[\left(\mu-\frac{\sigma^{2}}{2}\right) \tilde{t}_{i}+\frac{\sigma^{2}}{2} \sum_{j} \min \left(\tilde{t}_{i}, \tilde{t}_{j}\right)\right]} \mathrm{d} \tilde{t}_{1} \ldots \mathrm{d} \tilde{t}_{k} .
\end{aligned}
$$

Due to symmetry of integrand, we can order the integration variables as $\tilde{t}_{i}<\tilde{t}_{i+1}$, obtaining

$$
r_{k}(\mu, \sigma, t)=\Gamma(k+1) \int_{0}^{t} \int_{0}^{\tilde{t}_{k-1}} \ldots \int_{0}^{\tilde{t}_{2}} e^{\sum_{i=1}^{k} \mu \tilde{t}_{i}+\frac{\sigma^{2}}{2} \sum_{i \neq j ; i, j=1}^{k} \min \left(\tilde{t}_{i}, \tilde{t}_{j}\right)} \mathrm{d} \tilde{t}_{1} \ldots \mathrm{d} \tilde{t}_{k},
$$

whence

$$
\begin{aligned}
r_{k}(\mu, \sigma, t) & =\Gamma(k+1) \int_{0}^{t} \int_{0}^{\tilde{t}_{k-1}} \ldots \int_{0}^{\tilde{t}_{2}} e^{\sum_{i=1}^{k} \mu \tilde{t}_{i}+\sigma^{2} \sum_{i<j}^{n} \min \left(\tilde{t}_{i}, \tilde{t}_{j}\right)} \mathrm{d} \tilde{t}_{1} \ldots \mathrm{d} \tilde{t}_{k} \\
& =\Gamma(k+1) \int_{0}^{t} \int_{0}^{\tilde{t}_{k-1}} \cdots \int_{0}^{\tilde{t}_{2}} e^{\sum_{i=1}^{k} \mu \tilde{t}_{i}+\sigma^{2} \sum_{i=1}^{k}(k-i) \tilde{t}_{i}} \mathrm{~d} \tilde{t}_{1} \ldots \mathrm{d} \tilde{t}_{k} .
\end{aligned}
$$

After rearranging carefully the terms, we arrive at the final result:

$$
\begin{aligned}
r_{k}(\mu, \sigma, t)= & \Gamma(k+1) \int_{0}^{t} e^{\mu \tilde{t}_{k}} \int_{0}^{\tilde{t}_{k-1}} e^{\left(\mu+\sigma^{2}\right) \tilde{t}_{k-2}} \ldots \\
& \ldots \int_{0}^{\tilde{t}_{3}} e^{\left(\mu+(k-2) \sigma^{2}\right) \tilde{t}_{2}} \int_{0}^{\tilde{t}_{2}} e^{\left(\mu+(k-1) \sigma^{2}\right) \tilde{t}_{1}} \mathrm{~d} \tilde{t}_{1} \ldots \mathrm{d} \tilde{t}_{k} .
\end{aligned}
$$

Let us now expand further on eq. (16). It is convenient to first perform the rescaling $t_{i}=t u_{i}$. Then, by defining $\lambda_{j}=t\left(\mu+(k-j) \sigma^{2}\right)$, we obtain

$$
\begin{aligned}
r_{k}(\boldsymbol{\lambda}, t) & =t^{k} \Gamma(k+1) \int_{0}^{1} \mathrm{~d} u_{k} \int_{0}^{u_{k}} \mathrm{~d} u_{k-1} \ldots \int_{0}^{u_{2}} \mathrm{~d} u_{1} e^{\sum_{i=1}^{k} \lambda_{i} u_{i}} \\
& \equiv t^{k} \Gamma(k+1) s_{k}\left(\lambda_{1}, \ldots, \lambda_{k}\right) .
\end{aligned}
$$

Therefore, the computation of (9) reduces to the computation of

$$
s_{k}\left(\lambda_{1}, \ldots, \lambda_{k}\right)=\int_{0}^{1} \mathrm{~d} u_{k} \int_{0}^{u_{k}} \mathrm{~d} u_{k-1} \ldots \int_{0}^{u_{2}} \mathrm{~d} u_{1} e^{\sum_{i=1}^{k} \lambda_{i} u_{i}},
$$

a very similar formula had been obtained in [10]. Such relationship was also considered for the case of multiplicative noise diffusion processes [18-21], obtaining an analog recursion relationship. Given the importance of this result, we provide its proof. Such recursion relation has been observed in a variety of other works $[15,18]$. Given the importance of such recursion relation for our final result, we provide another proof of the recursion relationship in the following Lemma:

Lemma 2. For the quantity $s_{k}\left(\lambda_{1}, \ldots, \lambda_{k}\right)$, we have

$$
s_{k}\left(\lambda_{1}, \ldots, \lambda_{k}\right)=\frac{e^{\lambda_{k}} s_{k-1}\left(\lambda_{1}, \ldots, \lambda_{k-1}\right)-s_{k-1}\left(\lambda_{1}, \ldots \lambda_{k-2}, \lambda_{k-1}+\lambda_{k}\right)}{\lambda_{k}},
$$

with $s_{0}=1$.

Proof. Let us write

$$
\begin{aligned}
s_{k}\left(\lambda_{1}, \ldots, \lambda_{k}\right) & =\int_{0}^{1} \mathrm{~d} u_{k} \int_{0}^{u_{k}} \mathrm{~d} u_{k-1} \ldots \int_{0}^{u_{2}} \mathrm{~d} u_{1} e^{\sum_{i=1}^{k} \lambda_{i} u_{i}} \\
& =\int_{0}^{1} \mathrm{~d} u_{k} e^{\lambda_{k} u_{k}} f\left(u_{k}\right),
\end{aligned}
$$




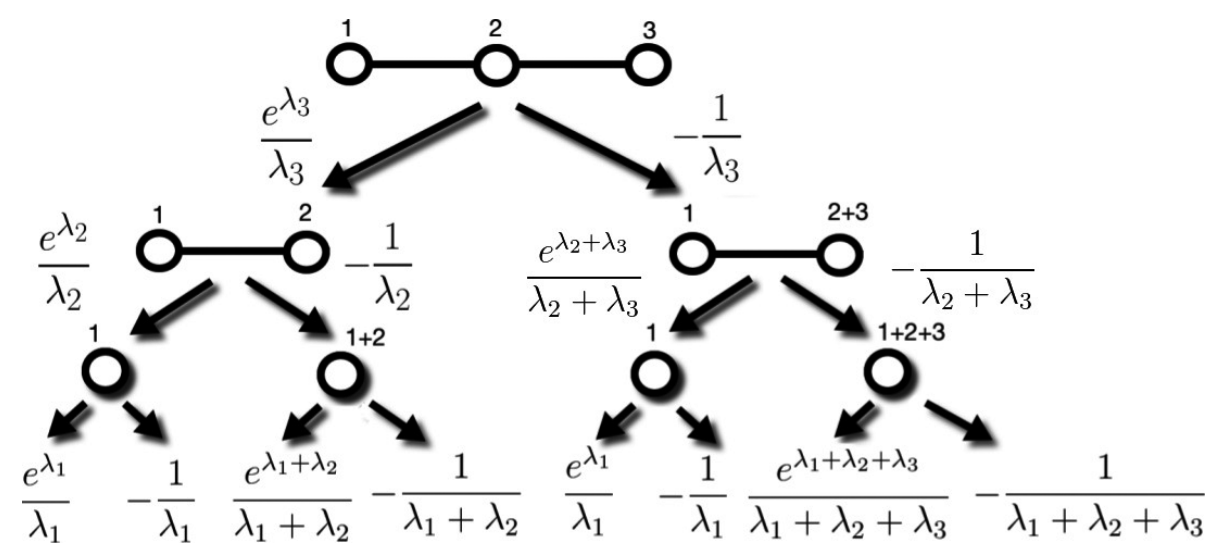

Fig. 1. Application of the graphical method for evaluating $k$-th moments of the exponential integrated Gaussian process to the case $k=3$.

with $f\left(u_{k}\right)=\int_{0}^{u_{k}} \mathrm{~d} u_{k-1} \cdots \int_{0}^{u_{2}} \mathrm{~d} u_{1} e^{\sum_{i=1}^{k-1} \lambda_{i} u_{i}}$. Integrating by parts,

$$
s_{k}\left(\lambda_{1}, \ldots, \lambda_{k}\right)=\left.\frac{e^{\lambda_{k} u_{k}}}{\lambda_{k}} f\left(u_{k}\right)\right|_{0} ^{1}-\int_{0}^{1} \mathrm{~d} u_{k} \frac{e^{\lambda_{k} u_{k}}}{\lambda_{k}} f^{\prime}\left(u_{k}\right),
$$

where

$$
\begin{aligned}
f^{\prime}\left(u_{k}\right) & =\int_{0}^{u_{k-1}} \cdots \int_{0}^{u_{2}} \mathrm{~d} u_{1} e^{\sum_{i=1}^{k-2} \lambda_{i} u_{i}+\lambda_{k-1} u_{k}} \\
& =e^{\lambda_{k-1} u_{k}} \int_{0}^{u_{k-1}} \cdots \int_{0}^{u_{2}} \mathrm{~d} u_{1} e^{\sum_{i=1}^{k-2} \lambda_{i} u_{i}} .
\end{aligned}
$$

Finally, the identity $f(0)=0$ gives the desired result.

Equation (20) suggests the evaluation of the averages by means of combinatorial considerations. A similar picture was shown in [18]. Indeed, the evaluation of the integral can proceed graphically, for any fixed order of the moment $k$, as in fig. 1. Starting from the top and using the properties of the recurrence relation, at each order $k$, we organize the recurrence on a binary tree. In fig. 1, each left branch will pull out a factor $e^{\lambda} / \lambda$, where $\lambda$ is the $\lambda$ obtained from the previous order. One has to consider the fact that however, in each right branch one pulls out a factor $-1 / \lambda$, and sums the two factors of $\lambda$ 's. To obtain the final formula, once the empty set has been reached, one multiplies the final term by all the factors in the branch.

We now give exact formulas for all the terms obtained from the recurrence. To lighten the notation, we define $\mu_{k, j}=\ll_{j}+\ldots+\ll_{k}$ and $s_{k}=s_{k}\left(\ll_{1}, \ldots, \ll_{k}\right)$. By iterating the second term of the recurrence, we obtain

$$
\begin{aligned}
s_{k}= & \frac{e^{\mu_{k, k}}}{\mu_{k, k}} s_{k-1}-\frac{e^{\mu_{k, k-1}}}{\mu_{k, k} \mu_{k, k-1}} s_{k-2}+\frac{1}{\mu_{k, k} \mu_{k, k-1}} s_{k-2}\left(\ll_{1}, \ldots, \ll_{k-3}, \mu_{k, k-2}\right) \\
= & \frac{e^{\mu_{k, k}}}{\mu_{k, k}} s_{k-1}-\frac{e^{\mu_{k, k-1}}}{\mu_{k, k} \mu_{k, k-1}} s_{k-2}+\frac{e^{\mu_{k, k-2}}}{\mu_{k, k} \mu_{k, k-1} \mu_{k, k-2}} s_{k-3} \\
& -\frac{1}{\mu_{k, k} \mu_{k, k-1} \mu_{k, k-2}} s_{k-3}\left(\ll_{1}, \ldots, \ll_{k-4}, \mu_{k, k-3}\right) \\
= & \ldots \\
= & \sum_{j=1}^{k}(-1)^{k-j} \frac{e^{\mu_{k, j}}}{\mu_{k, j} \mu_{k, j+1} \cdots \mu_{k, k}} s_{j-1}+(-1)^{k} \frac{1}{\mu_{k, 1} \ldots \mu_{k, k}} .
\end{aligned}
$$

Therefore,

$$
s_{k}\left(\ll_{1}, \ldots, \ll_{k}\right)=\sum_{j=1}^{k}(-1)^{k-j} \frac{e^{\ll_{j}+\ldots+\ll_{k}}}{\prod_{i=j}^{k}\left(\ll_{i}+\ldots+\ll_{k}\right)} s_{j-1}\left(\ll_{1}, \ldots, \ll_{j-1}\right)+\frac{(-1)^{k}}{\prod_{i=1}^{k}\left(\ll_{i}+\ldots+\ll_{k}\right)},
$$

which is a further improvement upon the recursion relation described before. In fact, now we have expanded the term which contained the "memory" part of the recursion.

The next Lemma is now an intermediate step towards evaluating the full recursion relationship. 
Lemma 3. Let $f_{k}=\sum_{j=0}^{k} e_{j}^{k} f_{j-1}$ with $f_{-1}=1$. Then

$$
f_{k}=\sum_{m=0}^{k} \sum_{0=i_{0}<i_{1}<\ldots<i_{m}<k+1=i_{m+1}} e_{i_{m}}^{i_{m+1}-1} e_{i_{m-1}}^{i_{m}-1} \ldots e_{i_{0}}^{i_{1}-1} .
$$

Proof. We proceed the proof by induction on $k$. We have $f_{k}=e_{0}^{0}$, which holds for $k=0$. We assume that the claim holds for $k$ and let us prove it for $k+1$. By the recurrence relation, we have

$$
f_{k+1}=e_{0}^{k+1}+\sum_{j=1}^{k+1} e_{j}^{k+1} f_{j-1} .
$$

If we now use the induction hypothesis, we find

$$
\begin{aligned}
f_{k+1} & =e_{0}^{k+1}+\sum_{j=1}^{k+1} e_{j}^{k+1}\left(\sum_{m=0}^{j-1} \sum_{0=i_{0}<i_{1}<\ldots<i_{m}<j=i_{m+1}} e_{i_{m}}^{j-1} e_{i_{m-1}}^{i_{m}-1} \ldots e_{i_{0}}^{i_{1}-1}\right) \\
& =e_{0}^{k+1}+\sum_{j=1}^{k+1}\left(\sum_{m=0}^{j-1} \sum_{0=i_{0}<i_{1}<\ldots<i_{m}<j=i_{m+1}<i_{m+2}=k+2} e_{i_{m+1}}^{i_{m+2}-1} e_{i_{m}}^{j-1} e_{i_{m}-1}^{i_{m}-1} \ldots e_{i_{0}}^{i_{1}-1}\right) \\
& =e_{0}^{k+1}+\sum_{m=1}^{k+1}\left(\sum_{j=m}^{k+1} e_{0=i_{0}<i_{1}<\ldots<i_{m}-1<j=i_{m}<i_{m+1}=k+2}^{i_{i_{m}+1}-1} e_{i_{m}-1}^{i_{m}-1} \ldots e_{i_{0}}^{i_{1}-1}\right) \\
& =e_{0}^{k+1}+\sum_{m=1}^{k+1} \sum_{0=i_{0}<i_{1}<\ldots<i_{m}<i_{m+1}=k+2}^{i_{i_{m}+1}-1} e_{i_{m-1}}^{i_{m}-1} \ldots e_{i_{0}}^{i_{1}-1} \\
& =\sum_{m=0}^{k+1} e_{0=i_{0}<i_{1}<\ldots<i_{m}<i_{m+1}=k+2}^{i_{i_{m}+1}-1} e_{i_{m-1}}^{i_{m}-1} \ldots e_{i_{0}}^{i_{1}-1},
\end{aligned}
$$

which completes the induction step.

If $e_{j}^{k}=(-1)^{k-j} \frac{e^{\ll_{j}+\ldots+\ll_{k}}}{\prod_{i=j}^{k}\left(\ll_{i}+\ldots+\ll_{k}\right)}$ and $e_{0}^{k}=\frac{(-1)^{k}}{\prod_{i=1}^{k}\left(\ll_{i}+\ldots+\ll_{k}\right)}$, then eq. (24) can be written as

$$
s_{k}\left(\ll_{1}, \ldots, \ll_{k}\right)=\sum_{j=1}^{k} e_{j}^{k} s_{j-1}\left(\ll_{1}, \ldots, \ll_{j-1}\right)+e_{0}^{k},
$$

which allows us now to perform the full combinatorial expansion. If we now apply Lemma 3, we obtain

$$
\begin{aligned}
s_{k}\left(\ll_{1}, \ldots, \ll_{k}\right) & =\sum_{m=0}^{k} \sum_{0=i_{0}<i_{1}<\ldots<i_{m}<k+1=i_{m+1}} e_{i_{m}}^{i_{m+1}-1} e_{i_{m-1}}^{i_{m}-1} \ldots e_{i_{0}}^{i_{1}-1} \\
& =\sum_{m=0}^{k} \sum_{0=i_{0}<i_{1}<\ldots<i_{m}<k+1=i_{m+1}} \frac{(-1)^{i_{1}-1}}{\prod_{j=1}^{i_{1}-1}\left(\ll_{j}+\ldots+\ll_{i_{1}-1}\right)} \prod_{\ell=1}^{m} \frac{(-1)^{i_{\ell+1}-i_{\ell}-1} e^{\ll_{i_{\ell}}+\ldots+\ll_{i_{\ell+1}-1}}}{\prod_{j=i_{\ell}}^{i_{\ell+1}-1}\left(\ll_{j}+\ldots+\ll_{i_{\ell+1}-1}\right)} \\
& =\sum_{m=0}^{k} \sum_{0=i_{0}<i_{1}<\ldots<i_{m}<k+1=i_{m+1}} \frac{(-1)^{k-m} e^{\ll_{i_{1}}+\ldots+\ll_{k}}}{\prod_{j=1}^{i_{1}-1}\left(\ll_{j}+\ldots+\ll_{i_{1}-1}\right) \prod_{\ell=1}^{m} \prod_{j=i_{\ell}}^{i_{\ell+1}-1}\left(\ll_{j}+\ldots+\ll_{i_{\ell+1}-1}\right)},
\end{aligned}
$$

which is one of the key results of our work, as this is in fact the solution of the recursion relation. We are now in the position of stating the first of the two main results of our paper, summarizing the expansion above into the following:

Theorem 1. For all $k \geq 0, s_{k}\left(\ll_{1}, \ldots, \ll_{k}\right)$ is given by

$$
\sum_{m=0}^{k} \sum_{0=i_{0}<i_{1}<\ldots<i_{m}<k+1=i_{m+1}} \frac{(-1)^{k-m} e^{\ll_{i_{1}}+\ldots+\ll_{k}}}{\prod_{j=1}^{i_{1}-1}\left(\ll_{j}+\ldots+\ll_{i_{1}-1}\right) \prod_{\ell=1}^{m} \prod_{j=i_{\ell}}^{i_{\ell+1}-1}\left(\ll_{j}+\ldots+\ll_{i_{\ell+1}-1}\right)} .
$$


It is often useful to understand what is the leading order in expansions of the form above. This is easily obtained from the theorem above. Let us consider the following expansion:

$$
\begin{aligned}
& s_{k}\left(t \ll_{1}, \ldots, t \ll_{k}\right)= \\
& \sum_{m=0}^{k} \sum_{0=i_{0}<i_{1}<\ldots<i_{m}<k+1=i_{m+1}} \frac{(-1)^{k-m} e^{t\left(\ll_{i_{1}}+\ldots+\ll_{k}\right)}}{t^{k-1} \prod_{j=1}^{i_{1}-1}\left(\ll_{j}+\ldots+\ll_{i_{1}-1}\right) \prod_{\ell=1}^{m} \prod_{j=i_{\ell}}^{i_{\ell+1}-1}\left(\ll_{j}+\ldots+\ll_{i_{\ell+1}-1}\right)} .
\end{aligned}
$$

Hence, the coefficient of $t^{n}$ in $s_{k}\left(t \ll_{1}, \ldots, t \ll_{k}\right)$ is given by

$$
\frac{1}{(n+k-1) !} \sum_{m=0}^{k} \sum_{0=i_{0}<i_{1}<\ldots<i_{m}<k+1=i_{m+1}} \frac{(-1)^{k-m}\left(\ll_{i_{1}}+\ldots+\ll_{k}\right)^{n+k-1}}{\prod_{j=1}^{i_{1}-1}\left(\ll_{j}+\ldots+\ll_{i_{1}-1}\right) \prod_{\ell=1}^{m} \prod_{j=i_{\ell}}^{i_{\ell+1}-1}\left(\ll_{j}+\ldots+\ll_{i_{\ell+1}-1}\right)} .
$$

The next result of our paper is merely the observation that the structure of the solution can be recast as a determinant equation. In fact, if we define $s_{k}=s_{k}\left(\ll_{1}, \ldots, \ll_{k}\right)$ for all $k \geq 0$, where we define $s_{0}=s_{-1}=1$. If $e_{j}^{k}=(-1)^{k-j} \frac{e^{\ll_{j}+\ldots+\ll_{k}}}{\prod_{i=j}^{k}\left(\ll_{i}+\ldots+\ll_{k}\right)}$ and $e_{0}^{k}=\frac{(-1)^{k}}{\prod_{i=1}^{k}\left(\ll_{i}+\ldots+\ll_{k}\right)}$, then eq. (24) can be written as

$$
s_{k}=\sum_{j=0}^{k} e_{j}^{k} s_{j-1}
$$

We are in place of stating the main result of this paper. A cleaner formula can be in fact obtained in terms of determinants. Using Theorem 4.20 in [22] (see also [23]), we obtain the following result:

Theorem 2. For all $k \geq 0, s_{k}=s_{k}\left(\ll_{1}, \ldots, \ll_{k}\right)$ is given by

$$
\operatorname{det}\left(\begin{array}{ccccccc}
e_{k}^{k} & e_{k-1}^{k} & e_{k-2}^{k} & \cdots & e_{2}^{k} & e_{1}^{k} & e_{0}^{k} \\
-1 & e_{k-1}^{k-1} & e_{k-2}^{k-1} & \ldots & e_{2}^{k-1} & e_{1}^{k-1} & e_{0}^{k-1} \\
0 & -1 & e_{k-2}^{k-2} & \ldots & e_{2}^{k-2} & e_{1}^{k-2} & e_{0}^{k-2} \\
\vdots & \vdots & \vdots & & \vdots & \vdots & \vdots \\
0 & 0 & 0 & & -1 & e_{1}^{1} & e_{0}^{1} \\
0 & 0 & 0 & & 0 & -1 & e_{0}^{0}
\end{array}\right)
$$

where $e_{j}^{k}=(-1)^{k-j} \frac{e^{\ll_{j}+\ldots+\ll_{k}}}{\prod_{i=j}^{k}\left(\ll_{i}+\ldots+\ll_{k}\right)}$ and $e_{0}^{k}=\frac{(-1)^{k}}{\prod_{i=1}^{k}\left(\ll_{i}+\ldots+\ll_{k}\right)}$.

Equation (26) is a new, determinantal representation for the moments of the integrated geometric Brownian motion. The emergence of a final expression in terms of a determinant is not surprising, a posteriori, if we consider the fact that these are common in the context of finite differences [16]. However, in order to convince ourselves that this equation reduces to well know expressions, let us elucidate the properties of eq. (26) by means of an example. Let us consider the case $n=3$ and $\sigma=0$, for which the stochastic differential equation reduces to ordinary logistic differential equation. In this case, we have an exact formula for $\lambda_{j}=\mu t$ :

$$
n=3: \operatorname{Det}\left(\left[\begin{array}{cccc}
\frac{e^{\mu t}}{\mu t} & -1 / 2 \frac{e^{2 \mu t}}{\mu^{2} t^{2}} & 1 / 6 \frac{e^{3 \mu t}}{\mu^{3} t^{3}} & -1 / 6 \frac{1}{\mu^{3} t^{3}} \\
-1 & \frac{e^{\mu t}}{\mu t} & -1 / 2 \frac{e^{2 \mu t}}{\mu^{2} t^{2}} & 1 / 2 \frac{1}{\mu^{2} t^{2}} \\
0 & -1 & \frac{e^{\mu t}}{\mu t} & -\frac{1}{\mu t} \\
0 & 0 & -1 & 1
\end{array}\right]\right)=1 / 6 \frac{\left(e^{\mu t}-1\right)^{3}}{\mu^{3} t^{3}},
$$

which provides the exact value obtained from the deterministic logistic equation.

The previous results are general enough to hold also for averages of the form

$$
\tilde{r}_{n}(\mu, \sigma, t)=\left\langle e^{\left(\mu-\frac{\sigma^{2}}{2}\right) t+\sigma W_{t}} F[W, t]^{n}\right\rangle=e^{\left(\mu-\frac{\sigma^{2}}{2}\right) t}\left\langle e^{\sigma W_{t}} F[W, t]^{n}\right\rangle .
$$



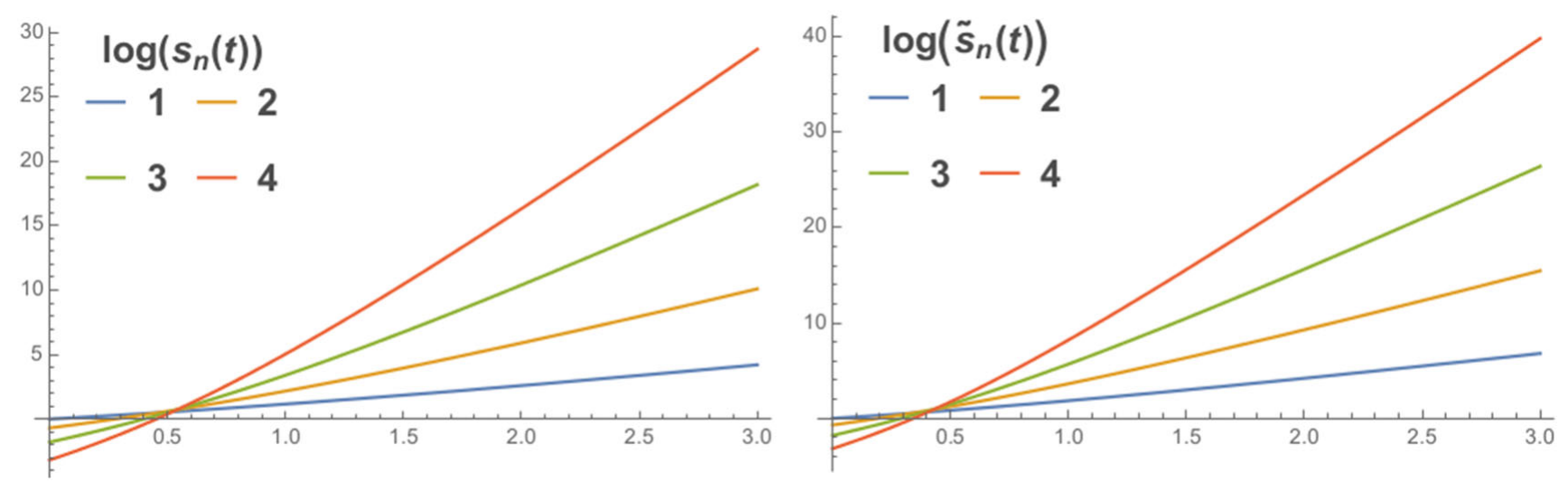

Fig. 2. Plot of the functions $s_{n}(\mu, \sigma, t)$ and $\tilde{s}_{n}(\mu, \sigma, t)$ provided in appendix A as a function of $t$ for $\mu=3, \sigma=1$ for $n=1,2,3,4$.

It is easy to see that eq. (26) applies to this case as well. In fact, we just need to substitute $\tilde{\lambda}_{k}=\lambda_{k}+t \sigma^{2}$ and multiply by a factor:

$$
\begin{aligned}
\tilde{r}_{n}(\mu, \sigma, t) & =e^{\left(\mu-\frac{\sigma^{2}}{2}\right) t} t^{n} e^{\frac{\sigma^{2}}{2} t} \Gamma(n+1) s_{n}\left(\tilde{\lambda}_{1}, \ldots, \tilde{\lambda}_{n}\right) \\
& =e^{\mu t} t^{n} \Gamma(n+1) s_{n}\left(\tilde{\lambda}_{1}, \ldots, \tilde{\lambda}_{n}\right) .
\end{aligned}
$$

The above expression will be used in the following section as an application to averages in the logistic stochastic differential equation. For completeness, the appendix contains the analytical values of the functions $s_{k}\left(\lambda_{1}, \ldots, \lambda_{k}\right)$ and $s_{k}\left(\tilde{\lambda}_{1}, \ldots, \tilde{\lambda}_{k}\right)$ for the cases $n \leq 4$. It is immediate that, when $t \approx 0$, we have

$$
s_{n}\left(t \mu, t\left(\mu+\sigma^{2}\right), \ldots, t\left(\mu+(n-1) \sigma^{2}\right)\right) \approx \frac{1}{t^{n}} .
$$

This fact implies that eq. (28) is analytic in $t=0$. Figure 2 is a plot of $s_{n}(t)$ for $\mu=1, \sigma=0.1$.

\section{Averages of Logistic SDE in perturbation theory}

As an application of the formula (28), we focus on the solution of the logistic stochastic differential equation,

$$
\mathrm{d} x=x\left[\mu\left(1-\frac{x}{\tilde{x}}\right) \mathrm{d} t+\sigma \mathrm{d} W\right],
$$

given by (we follow $[14,24]$ )

$$
\begin{aligned}
x(t) & =x_{0} e^{\left(\mu-\frac{\sigma^{2}}{2}\right) t+\sigma W_{t}}\left(1+\frac{\mu x_{0}}{\tilde{x}} \int_{0}^{t} e^{\left(\mu-\frac{\sigma^{2}}{2}\right) s+\sigma W_{s}} \mathrm{~d} s\right)^{-1} \\
& =x_{0} e^{\left(\mu-\frac{\sigma^{2}}{2}\right) t+\sigma W_{t}} \sum_{n=0}^{\infty}(-1)^{n}\left(\frac{\mu x_{0}}{\tilde{x}} \int_{0}^{t} e^{\left(\mu-\frac{\sigma^{2}}{2}\right) s+\sigma W_{s}} \mathrm{~d} s\right)^{n} .
\end{aligned}
$$

We evaluate the average of the solution $x(t)$,

$$
\begin{aligned}
\langle x(t)\rangle & =\left\langle x_{0} e^{\left(\mu-\frac{\sigma^{2}}{2}\right) t+\sigma W_{t}} \sum_{n=0}^{\infty}(-1)^{n}\left(\frac{\mu x_{0}}{\tilde{x}} \int_{0}^{t} e^{\left(\mu-\frac{\sigma^{2}}{2}\right) s+\sigma W_{s}} \mathrm{~d} s\right)^{n}\right\rangle \\
& =x_{0} \sum_{n=0}^{\infty}(-1)^{n}\left(\frac{\mu x_{0}}{\tilde{x}}\right)^{n}\left\langle e^{\left(\mu-\frac{\sigma^{2}}{2}\right) t+\sigma W_{t}}\left(\int_{0}^{t} e^{\left(\mu-\frac{\sigma^{2}}{2}\right) s+\sigma W_{s}} \mathrm{~d} s\right)^{n}\right\rangle \\
& =x_{0} e^{\mu t} \sum_{n=0}^{\infty}(-1)^{n}\left(t \frac{\mu x_{0}}{\tilde{x}}\right)^{n} \Gamma(n) s_{n}\left(\tilde{\lambda}_{1}, \ldots, \tilde{\lambda}_{n}\right),
\end{aligned}
$$




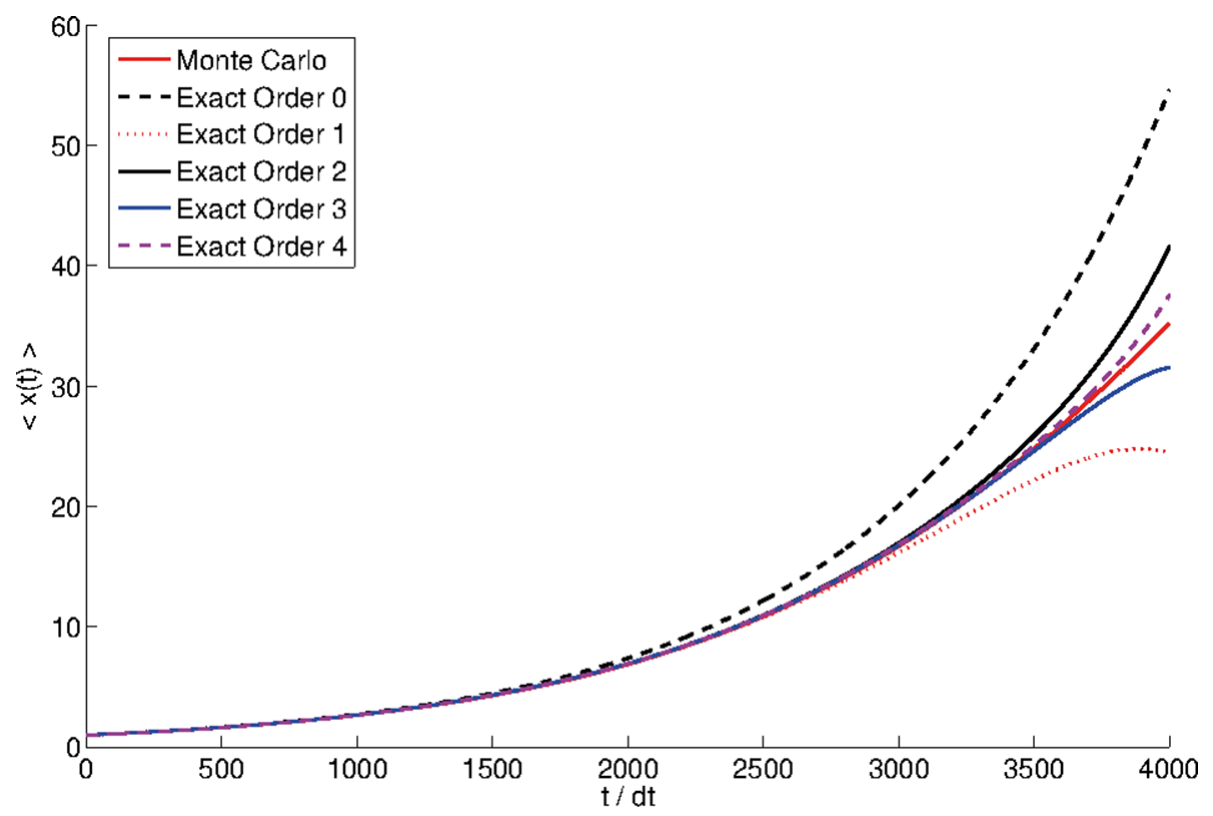

Fig. 3. Plot of the mean of solution of the logistic stochastic differential equations for $\mu=1, \sigma=0.1, \tilde{x}=100$, and with $\mathrm{d} t=10^{-3}$, solved numerically using a stochastic Euler method and averaged over 1000 simulations (solid red), versus the analytical solution obtained at $k$-th order, considering $\xi=\frac{x_{0}}{\tilde{x}}$ the perturbative parameter. We can observe that at higher order we obtain a solution closer to the one simulated.

and observe that it involves the moments of (28). In the limit $\tilde{x} \rightarrow \infty$, eq. (32) reduces to the average of the geometric Brownian motion. We consider now truncations of the mean of $x(t)$ at $k$-th order, $\langle x(t)\rangle_{k}=$ $x_{0} e^{\mu t} \sum_{n=0}^{k}(-1)^{n}\left(t \frac{\mu x_{0}}{\tilde{x}}\right)^{n} \Gamma(n) s_{n}\left(\tilde{\lambda}_{1}, \ldots, \tilde{\lambda}_{n}\right)$, and compare the truncated solution to the one obtained numerically. In fig. 3, we plot $\langle x(t)\rangle_{k}$ for $k=0,1, \ldots, 4$ obtained for $\mu=1, \sigma=0.1, \tilde{x}=100$ by means of a stochastic Euler method with $\mathrm{d} t=10^{-3}$, and the averages obtained using Monte Carlo over 1000 samples. We observe that the higher the order of the approximation, the closer we are to the solution obtained by Monte Carlo ${ }^{1}$.

\section{Conclusions}

In this paper, we have presented new exact formulas for the moments of the integrated exponential Brownian motion in terms of sums and determinants, and based on recent results obtained in [23]. We described a simple graphical method to evaluate them, based on a recurrence relation. In this paper however, we have taken an alternative route based on combinatorics. Exact formulas were proved in [10] in terms of polynomials, and in [18] using the same recursion relation we use in this work. After realizing that the mean can be evaluated exactly using the properties of Gaussian integrals, and after observing that these moments feature a recurrence relation, we have shown that exact expressions can be obtained via a combinatorial argument. These new exact expressions were then observed to be equivalent to evaluating the determinant of a specific linear operator which depends on the order of the moment to be evaluated.

Our result is in line with work currently done by several authors in order to elucidate the importance of market microstructure, and in particular of a carrying capacity, which can be seen as a transaction cost proportional to the bid volume. For instance, transaction costs are well known in the literature [25]. For the case of market impact, studies have been put forward in [26], and more precise statements based on data were made more recently in [12,13,27]. Our results are relevant in particular in light of the recent interest concerning the importance of ergodicity breaking in financial time series, for instance in the case of the Geometric Brownian Motion in [28], and for optimal trading strategies using the Kelly criterion in [29]. These results were recently generalized to the case in which one has linear transaction costs in [14]. There, in particular, it has been shown that in order to obtain optimal trading strategies, due to the time asymmetry of the process, each investor has to optimize along his own trading strategy, obtaining a closed form solution for the optimal leverage. Obtaining these optimal solutions involves evaluating perturbatively the average of solutions of logistic stochastic differential equation, and it is clear that the application of our exact moment equations is of extreme relevance.

In order to convince further the reader of our formula in terms of the determinant, we have applied the obtained formulas to the exact solution of the logistic stochastic differential equation. There, the evaluation of the ensemble

\footnotetext{
1 The numerical integration of the differential equation is performed by a stochastic Euler method.
} 
expectation values of certain observables can be carried out with our method, via Taylor expansion. In particular, the comparison of the mean solution obtained by means of Monte Carlo simulations with our method shows that our formula allows to approximate to a higher precision some ensemble averages of properties of the solution of the stochastic differential equation.

To conclude, in this we work we observed that the recursion relation can be casted into the framework of graph reductions, and if applied on a chain graph provides insights for the solution of the logistic stochastic differential equation. This property was observed to be a special case of a more general mathematical structure in [30], a generalized Tutte polynomial. The Tutte polynomial is a graph invariant which has several applications in statistical physics being connected to the partition function of the q-Potts model; this is in fact an active area of research also in quantum field theory, since generalized and non-commutative Tutte polynomials are connected to scattering amplitudes. Further work is necessary to understand whether such accidental mapping can turn to be a fruitful line of research.

SS is supported by the Royal Society and EPSRC.

\section{Appendix A. Exact formulas for $n \leq 4$}

Below we report the functions $s_{k}(\mu, \sigma, t) \equiv s_{k}\left(\lambda_{1}, \ldots, \lambda_{k}\right)$ and $\tilde{s}_{k}(\mu, \sigma, t) \equiv s_{k}\left(\tilde{\lambda}_{1}, \ldots, \tilde{\lambda}_{k}\right)$ up to $k=4$ :

$$
\begin{aligned}
& s_{1}(\mu, \sigma, t)=\frac{e^{\mu t}-1}{\mu t} ; \quad s_{2}(\mu, \sigma, t)=\frac{\frac{e^{t\left(2 \mu+\sigma^{2}\right)}-1}{2 \mu+\sigma^{2}}+\frac{1-e^{\mu t}}{\mu}}{t^{2}\left(\mu+\sigma^{2}\right)} \\
& s_{3}(\mu, \sigma, t)=\frac{2 \mu^{2}\left(e^{3 t\left(\mu+\sigma^{2}\right)}-3 e^{t\left(2 \mu+\sigma^{2}\right)}+3 e^{\mu t}-1\right)+6 \sigma^{4}\left(e^{\mu t}-1\right)+\mu \sigma^{2}\left(e^{3 t\left(\mu+\sigma^{2}\right)}-9 e^{t\left(2 \mu+\sigma^{2}\right)}+15 e^{\mu t}-7\right)}{3 \mu t^{3}\left(\mu+\sigma^{2}\right)\left(2 \mu+\sigma^{2}\right)\left(\mu+2 \sigma^{2}\right)\left(2 \mu+3 \sigma^{2}\right)} ; \\
& s_{4}(\mu, \sigma, t)=\frac{3 \mu^{2} \sigma^{2}\left(-8 e^{3 t\left(\mu+\sigma^{2}\right)}+18 e^{t\left(2 \mu+\sigma^{2}\right)}+e^{4 \mu t+6 \sigma^{2} t}-16 e^{\mu t}+5\right)-30 \sigma^{6}\left(e^{\mu t}-1\right)}{6 \mu t^{4}\left(\mu+\sigma^{2}\right)\left(2 \mu+\sigma^{2}\right)\left(\mu+2 \sigma^{2}\right)\left(\mu+3 \sigma^{2}\right)\left(2 \mu+3 \sigma^{2}\right)\left(2 \mu+5 \sigma^{2}\right)} \\
& +\frac{2 \mu^{3}\left(-4 e^{3 t\left(\mu+\sigma^{2}\right)}+6 e^{t\left(2 \mu+\sigma^{2}\right)}+e^{4 \mu t+6 \sigma^{2} t}-4 e^{\mu t}+1\right)}{6 \mu t^{4}\left(\mu+\sigma^{2}\right)\left(2 \mu+\sigma^{2}\right)\left(\mu+2 \sigma^{2}\right)\left(\mu+3 \sigma^{2}\right)\left(2 \mu+3 \sigma^{2}\right)\left(2 \mu+5 \sigma^{2}\right)} \\
& +\frac{\mu \sigma^{4}\left(-10 e^{3 t\left(\mu+\sigma^{2}\right)}+54 e^{t\left(2 \mu+\sigma^{2}\right)}+e^{4 \mu t+6 \sigma^{2} t}-82 e^{\mu t}+37\right)}{6 \mu t^{4}\left(\mu+\sigma^{2}\right)\left(2 \mu+\sigma^{2}\right)\left(\mu+2 \sigma^{2}\right)\left(\mu+3 \sigma^{2}\right)\left(2 \mu+3 \sigma^{2}\right)\left(2 \mu+5 \sigma^{2}\right)} ; \\
& \tilde{s}_{1}(\mu, \sigma, t)=\frac{e^{t\left(\mu+\sigma^{2}\right)}-1}{t\left(\mu+\sigma^{2}\right)} ; \quad \tilde{s}_{2}(\mu, \sigma, t)=\frac{\frac{e^{2 \mu t+3 \sigma^{2} t}-1}{2 \mu+3 \sigma^{2}}-\frac{e^{t\left(\mu+\sigma^{2}\right)}-1}{\mu+\sigma^{2}}}{t^{2}\left(\mu+2 \sigma^{2}\right)} ; \\
& \tilde{s}_{3}(\mu, \sigma, t)=\frac{-\frac{\mu+3 \sigma^{2}}{2 \mu^{2}+5 \mu \sigma^{2}+3 \sigma^{4}}+\left(\frac{2}{\mu+\sigma^{2}}+\frac{1}{-2 \mu-5 \sigma^{2}}\right) e^{t\left(\mu+\sigma^{2}\right)}-\frac{3 e^{2 \mu t+3 \sigma^{2} t}}{2 \mu+3 \sigma^{2}}+\frac{e^{3 t\left(\mu+2 \sigma^{2}\right)}}{2 \mu+5 \sigma^{2}}}{3 t^{3}\left(\mu+2 \sigma^{2}\right)\left(\mu+3 \sigma^{2}\right)} \\
& \tilde{s}_{4}(\mu, \sigma, t)=\frac{\frac{2}{2 \mu^{2}+9 \mu \sigma^{2}+10 \sigma^{4}}-\frac{1}{4 \mu^{2}+24 \mu \sigma^{2}+35 \sigma^{4}}-\frac{6\left(\mu+4 \sigma^{2}\right)}{4 \mu^{3}+28 \mu^{2} \sigma^{2}+61 \mu \sigma^{4}+42 \sigma^{6}}+\frac{2\left(\mu+4 \sigma^{2}\right)}{\left(\mu+\sigma^{2}\right)\left(\mu+2 \sigma^{2}\right)\left(2 \mu+5 \sigma^{2}\right)}}{6 t^{4}\left(\mu+3 \sigma^{2}\right)\left(\mu+4 \sigma^{2}\right)} \\
& +\frac{e^{t\left(\mu+\sigma^{2}\right)}\left(-\frac{2\left(\mu+4 \sigma^{2}\right)}{\left(\mu+\sigma^{2}\right)\left(\mu+2 \sigma^{2}\right)\left(2 \mu+5 \sigma^{2}\right)}-\frac{2 e^{t\left(2 \mu+5 \sigma^{2}\right)}}{2 \mu^{2}+9 \mu \sigma^{2}+10 \sigma^{4}}+\frac{e^{3 t\left(\mu+3 \sigma^{2}\right)}}{4 \mu^{2}+24 \mu \sigma^{2}+35 \sigma^{4}}+\frac{6\left(\mu+4 \sigma^{2}\right) e^{t\left(\mu+2 \sigma^{2}\right)}}{\left(\mu+2 \sigma^{2}\right)\left(2 \mu+3 \sigma^{2}\right)\left(2 \mu+7 \sigma^{2}\right)}\right)}{6 t^{4}\left(\mu+3 \sigma^{2}\right)\left(\mu+4 \sigma^{2}\right)} .
\end{aligned}
$$

Open Access This is an open access article distributed under the terms of the Creative Commons Attribution License (http://creativecommons.org/licenses/by/4.0), which permits unrestricted use, distribution, and reproduction in any medium, provided the original work is properly cited. 


\section{References}

1. C. Gardiner, Stochastic Methods (Springer-Verlag, Berlin, 2009).

2. M. Yor, Exponential Functionals of Brownian Motion and Related Processes (Springer-Verlag, Berlin, 2001).

3. J.P. Bouchaud, M. Potters, Theory of Financial Risks (Cambridge University Press, Cambridge, 2000).

4. D.J. Wilkinson, Stochastic Modelling for Systems Biology (CRC Press, New York, 2012).

5. H. Matsumoto, M. Yor, Prob. Surv. 2, 312 (2005).

6. H. Matsumoto, M. Yor, Prob. Surv. 2, 348 (2005).

7. D. Dufresne, Adv. Appl. Prob. 33, 223 (2001).

8. C. Monthus, A. Comtet, J. Phys. A 4, 635 (1994).

9. A. Comtet, C. Monthus, J. Phys. A 29, 1331 (1996).

10. M. Yor, Adv. Appl. Prob. 24, 509 (1992).

11. I.V. Girsanov, Theory Probab. Appl. 5, 285 (1960).

12. E. Moro, J. Vicente, L.G. Moyano, A. Gerig, J.D. Farmer, G. Vaglica, F. Lillo, R.N. Mantegna, Phys. Rev. E 80, 066102 (2009).

13. E. Zarinelli, M. Treccani, J.D. Farmer, F. Lillo, Mark. Microstruct. Liq. 1, 1 (2015).

14. F. Caravelli, L. Sindoni, F. Caccioli, C. Ududec, arXiv:1510.05123.

15. B.J.C. Baxter, R. Brummelhuis, J. Comput. Appl. Math. 236, 424 (2011).

16. C. De Boor, Surv. Approx. Theory 1, 46 (2005).

17. M. Salmhofer, Renormalization - An Introduction (Springer-Verlag, Berlin, 1999).

18. D. Delpini, Modeling and Analysis of Financial Time Series beyond Geometric Brownian Motion, PhD Thesis, Università degli Studi di Pavia (2010).

19. G. Bormetti, D. Delpini, Phys. Rev. E 81, 032102 (2010).

20. D. Delpini, G. Bormetti, Phys. Rev. E 83, 041111 (2011).

21. D. Delpini, G. Bormetti, Quant. Financ. 15, 15971608 (2015).

22. R. Vein, P. Dale, Determinants and Their Applications in Mathematical Physics (Springer-Verlag, Berlin, 1999).

23. M. Janjić, J. Integer Seq. 15, 12.3 .5 (2012).

24. P.E. Kloeden, E. Platen, Numerical Solution of Stochastic Differential Equations (Springer Science and Business Media, 2013).

25. R.C. Merton, J. Econ. Theory 3, 373 (1971).

26. J.D. Farmer, L. Gillemot, F. Lillo, S. Mike, A. Sen, Quant. Financ. 4, 383 (2004).

27. S. Ciliberti, J. Bouchaud, M. Potters, Wilmott J. 1, 87 (2009).

28. O. Peters, Quant. Financ. 11, 1593 (2011).

29. E.O. Thorp, in Handbook of Asset and Liability Management: Theory and Methodology (Elsevier, 2006).

30. J. Ben Geloun, F. Caravelli, arXiv:1512.02278. 